\title{
The relation between body semantics and spatial body representations
}

\author{
Michiel van Elk*, Olaf Blanke \\ Laboratory of Cognitive Neuroscience, Brain Mind Institute, École Polytechnique Fédérale de Lausanne, Switzerland
}

\section{A R T I C L E I N F O}

\section{Article history:}

Received 11 July 2011

Received in revised form 6 September 2011

Accepted 6 September 2011

Available online 28 September 2011

\section{PsycINFO classification:}

2340

2720

Keywords:

Body semantics

Embodied cognition

Symbolic distance effect

Body representation

Iconicity judgment

Visual field

\begin{abstract}
A B S T R A C T
The present study addressed the relation between body semantics (i.e. semantic knowledge about the human body) and spatial body representations, by presenting participants with word pairs, one below the other, referring to body parts. The spatial position of the word pairs could be congruent (e.g. EYE / MOUTH) or incongruent (MOUTH / EYE) with respect to the spatial position of the words' referents. In addition, the spatial distance between the words' referents was varied, resulting in word pairs referring to body parts that are close (e.g. EYE / MOUTH) or far in space (e.g. EYE / FOOT). A spatial congruency effect was observed when subjects made an iconicity judgment (Experiments 2 and 3 ) but not when making a semantic relatedness judgment (Experiment 1). In addition, when making a semantic relatedness judgment (Experiment 1 ) reaction times increased with increased distance between the body parts but when making an iconicity judgment (Experiments 2 and 3 ) reaction times decreased with increased distance. These findings suggest that the processing of body-semantics results in the activation of a detailed visuo-spatial body representation that is modulated by the specific task requirements. We discuss these new data with respect to theories of embodied cognition and body semantics.
\end{abstract}

(C) 2011 Elsevier B.V. All rights reserved.

\section{Introduction}

Imagine the following simple experiment. Show a four-year-old boy the picture of an airplane and ask him to indicate the position of the eyes and arms of the plane. Probably without problems he will point to respectively the cockpit and the wings. As the example illustrates, young children already have a clear representation of the spatial relation between different body parts and the words referring to these body parts. In addition, children can easily transfer this knowledge to other animals and even artifacts as the example showed, via a process referred to as analogical reasoning (Gentner, 1977). Developmental studies indicate that the ability to identify body parts in response to verbal cues gradually emerges during the second year of life (Slaughter \& Heron, 2004; Witt, Cermak, \& Coster, 1990) and goes hand in hand with the acquisition of an abstract visuo-spatial representation of the human body (Buxbaum \& Coslett, 2001).

The strong association between body words and space is abundant in our everyday language as well, as evidenced for instance by expressions like 'from top to toe', 'at arm's length', or 'up to one's knees'. Although usually body words and space are strongly linked, in some cases this association can break down and body knowledge

\footnotetext{
* Corresponding author at: Laboratory of Cognitive Neuroscience, Brain Mind Institute, École Polytechnique Fédérale de Lausanne, Station 19, AI 2101, 1015 Lausanne, Switzerland. Tel.: +4121693 1771; fax: +41216931770.

E-mail address: michiel.vanelk@epfl.ch (M. van Elk).
}

can be selectively impaired, at either a visuo-spatial or a semantic level. For instance, an impairment in the visuo-spatial representation of the human body can be observed in patients characterized by auto/ heterotopagnosia, who often display difficulties with pointing to named body parts on oneself, on the body of another person or on a line drawing (Felician, Ceccaldi, Didic, Thinus-Blanc, \& Poncet, 2003; Sirigu, Grafman, Bressler, \& Sunderland, 1991). Interestingly, such deficits appear to be unrelated to a general deficit in spatial abilities or in semantic naming difficulties, as evidenced by a spared performance when animals or manmade object parts are used instead. Thereby, this patients' behavior stands in contrast with the difficulties experienced by patients with a specific loss of body semantics (Coslett, Saffran, \& Schwoebel, 2002; Kemmerer \& Tranel, 2008; Laiacona, Allamano, Lorenzi, \& Capitani, 2006; Schwoebel, Buxbaum, \& Coslett, 2004; Schwoebel \& Coslett, 2005; Shelton, Fouch, \& Caramazza, 1998; Suzuki, Yamadori, Fuji, \& Abe, 1997). These patients are unable to understand the meaning of words referring to body parts and to relate a word referring to a body part to its referent. Based on these findings it has been suggested that body semantics, i.e. semantic knowledge of the human body, comprises a distinct conceptual category that can be selectively impaired or spared (Coslett et al., 2002).

Recent studies suggest that body semantics are strongly linked to a spatial representation of the human body, containing veridical information about the relative distance between body parts (Smeets, Klugkist, Rooden, Anema, \& Postma, 2009; Struiksma, Noordzij, \& Postma, 2011). In these experiments subjects were asked to compare 
the relative distance between different pairs of body parts (e.g. compare the distance between one's ears to the distance between one's hips). It was found that reaction times decreased and accuracy increased with increasing physical distance differences between the body pairs. For instance, subjects responded faster and more accurately when comparing body parts with a large distance difference (e.g. 'ear-ear' vs. 'hip-hip') than when comparing body pairs with a small distance difference (e.g. 'shoulder-shoulder' vs. 'hip-hip'). In addition, in a property verification study it was found that subjects responded faster to body part items that were preceded by body parts in close compared to far spatial proximity (Struiksma et al., 2011). The authors suggest that subjects likely accessed a veridical spatial representation of the human body when processing body semantics, both in a distance comparison task and in a property verification task.

These findings have been interpreted as evidence for an embodied approach to cognition, according to which word meaning is grounded in modality-specific representations (for review, see: Borghi \& Cimatti, 2010; Fischer \& Zwaan, 2008). Further support for this view is found for instance in behavioral studies, showing that participants responded faster in a property verification task if the preceding word represented properties from the same instead of a different sensory modality (e.g., gustatory, auditory, or visual; Pecher, Zeelenberg, \& Barsalou, 2003). Neuroimaging studies have extended these data and provided support for the notion that the reading of words is accompanied by activation in modality-specific brain areas, as evidenced by the finding that the reading of action verbs or words referring to manipulable objects results in the activation of motor-related brain areas (e.g. Pulvermuller, 2005), whereas the processing of words for which visual properties are a defining feature (e.g. animals or fruits) results in the activation of visual brain regions (Martin, Wiggs, Ungerleider, \& Haxby, 1996). Similarly, when processing words referring to body parts, participants probably rely on a spatial representation of the human body, as evidenced by the finding of a distance effect for body parts that are further away as compared to those that are closer in physical space (cf. Smeets et al., 2009; Struiksma et al., 2011).

However, two as yet unresolved issues with respect to the relation between body semantics and spatial body representations are the following. First, it is unclear whether the spatial body representation contains only information about the relative position of different body parts (e.g. the relative distance between one's nose and one's belly button; cf. Smeets et al., 2009; Struiksma et al., 2011) or about the absolute position of body parts as well (e.g. knowing that in an upright position one's nose is located above one's belly button). Several studies have shown that humans have an internal model of gravity, resulting in a visual processing advantage for bodies that are aligned with the observer's body axis (Reed, Stone, Bozova, \& Tanaka, 2003; Westhoff \& Troje, 2007) and in effects of the observer's body orientation on the visual judgment of human bodies (Lobmaier \& Mast, 2007; Lopez, Bachofner, Mercier, \& Blanke, 2009). Similarly, it could well be that the processing of body semantics is accompanied by the activation of a spatial body representation, specifying the absolute position of body parts-as if the body is viewed from a $3 \mathrm{rd}$ person perspective.

Second, it remains to be determined how fine-grained the spatial body representation is that is activated upon reading words referring to body parts. Previous studies on body semantics have investigated the relation between different body parts only at a coarse level (i.e. small vs. large distances). However, patient studies indicate that the spatial body representation can be impaired at a very specific level, as evidenced for instance by patients with prosopagnosia, who are specifically impaired in their knowledge about the spatial relation between face parts (Barton, 2003), or patients with finger agnosia, who are no longer able to identify their individual fingers (Rusconi, Pinel, Dehaene, \& Kleinschmidt, 2010). Based on these findings, it is to be expected that body semantics are strongly linked to a detailed visuo-spatial body representation, specifying not only the relation between body parts that belong to a different category (i.e. the partonomy of head, trunk, arm and leg; Andersen, 1978), but that are part of the same category as well (e.g. knowing that the mouth is closer to the nose than to the eyes).

To address these issues, we employed an experimental paradigm that combined two previously well-established findings, namely the spatial congruency effect and the symbolic distance effect, and applied it to the domain of body semantics. Several studies have shown a spatial congruency effect for word pairs that are presented in a spatial position that is congruent to the actual position of the words' referents (also referred to as 'an iconic relationship between words and their meaning'; cf. Louwerse \& Jeuniaux, 2010; Zwaan \& Yaxley, 2003a, 2003b). For instance, subjects are faster to identify if two words belong to the same semantic category if the words are presented in a congruent spatial position (e.g. the word 'attic' presented above 'basement'), compared to an incongruent spatial position (e.g. the word 'basement' presented above 'attic'). Similarly, when applied to the domain of body semantics subjects may be faster in identifying body parts presented in a congruent compared to an incongruent spatial position. Such a finding would provide evidence for the notion that in addition to having a spatial representation of the relative distance between body parts (Struiksma et al., 2011), humans use their implicit knowledge about the absolute position of body parts as well, when processing body semantics.

In addition, previous studies have shown that when subjects are asked to estimate which is the larger of two items, their response times decrease as the difference between the sizes increases, which is known as the symbolic distance effect (Moyer \& Bayer, 1976; Moyer \& Landauer, 1967). Symbolic distance effects have been found when participants compared the sizes of familiar objects from memory (Dean, Dewhurst, Morris, \& Whittaker, 2005; Marschark, 1983; McGonigle \& Chalmers, 1984; Paivio, 1975) or the relative distance between body parts (Smeets et al., 2009; Struiksma et al., 2011) and these findings have been interpreted as evidence for the involvement of analog representations in visual imagery, by showing that the same principles that apply to visual processing apply to visual imagery. On the other hand, studies using the image scanning paradigm have shown that the mental scanning time across a visual image of a spatial configuration increases with increased distance between two points in the configuration (Borst, Kosslyn, \& Denis, 2006; Iachini \& Giusberti, 1996; Kosslyn, Ball, \& Reiser, 1978). An intriguing question is if subjects implicitly retrieve detailed information about the relative distance between body parts, when making a semantic categorization on words referring to body parts or when making a judgment about the spatial representation of the human body. In other words, in the present study we investigated whether response times to words referring to body parts were modulated by the spatial proximity of the body parts and if so, if the pattern would be consistent with the mental scanning hypothesis or the symbolic distance effect.

Thus, in the present study subjects were presented with word pairs referring to body parts. The spatial position of the word pairs could be congruent (e.g. eye above mouth: EYE/MOUTH) or incongruent (MOUTH/EYE) with respect to the spatial position of the words' referents. In addition, we manipulated at a fine-grained level the spatial distance on a human body that exists between the words' referents (body distance), resulting in word pairs referring to body parts that are close (e.g. EYE/MOUTH) or far in space (e.g. EYE/FOOT). These stimuli were used in three separate experiments to investigate under what conditions detailed spatial information about the human body is activated when processing body semantics.

In the first experiment, following Zwaan and Yaxley (2003a, 2003b), subjects were required to make a semantic relatedness judgment in response to the word pairs presented on the screen, by deciding whether both words belonged to the same semantic category (animals vs. body parts) or not. Although subjects did not explicitly make a distance comparison, it could well be that during a semantic categorization task 
(Experiment 1 ) subjects implicitly activate a visual representation of the human body to determine whether both words are part of the same semantic category. The use of a visual representation to determine if two words are part of the same object should be reflected in an increase in reaction times with increased distance between body parts, conform the mental scanning hypothesis (Borghi, Glenberg, \& Kaschak, 2004; Borst et al., 2006; Iachini \& Giusberti, 1996; Kosslyn et al., 1978).

Previous studies have shown that visuo-spatial effects during language processing are strongly driven by task requirements (Louwerse \& Jeuniaux, 2008, 2010). For instance, paying attention to the actionrelated properties of objects (e.g. a tennis ball, a hammer) resulted in increased activation in motor-related brain areas, whereas attending to the visual properties of these same objects has been associated with increased visual activation (van Dam et al., in press). To study the top-down effects of task requirements on the relation between body semantics and spatial body representations, in the 2nd and 3rd experiment subjects performed an iconicity judgment task, deciding whether the spatial position of words referring to body parts on the screen was congruent or incongruent with respect to the position of the real-world referents of these words. Whereas in the first experiment the visual perspective was left implicit, in the second experiment the iconicity judgment required subjects to represent human bodies as viewed from a 3rd person perspective. This manipulation likely results in the activation of information about the absolute rather than the relative spatial position of body parts and as a consequence, in the second experiment we expected a spatial congruency effect, reflected in faster reaction times for body words represented in a congruent compared to an incongruent spatial position. The iconicity judgment task requires a comparison between an observed spatial arrangement (i.e. the words on the screen) and a memorized spatial arrangement (i.e. one's spatial body representation). Please note that the distance difference between the words on the screen remained constant (i.e. small), while the distance of the memorized spatial arrangement varied. As a consequence the implicit comparison between the observed and the memorized spatial representation could result in a symbolic distance effect, reflected in faster reaction times for body parts that are far in space (Moyer \& Bayer, 1976).

Finally, previous studies have shown a hemispheric specialization for spatial iconicity effects (Zwaan \& Yaxley, 2003a) and for the processing of body semantics (Coslett et al., 2002; Laiacona et al., 2006; Schwoebel \& Coslett, 2005). To investigate hemispheric specialization with respect to the processing of body semantics, in a third experiment subjects performed an iconicity judgment task while words were presented to the left and right visual field.

\section{Experiment 1: Semantic relatedness judgment}

\subsection{Methods}

\subsubsection{Participants}

In the first experiment 21 subjects participated ( 8 females, mean age $=20.6$ years $)$, who received a financial remuneration for participation. Subjects declared themselves through informal verbal inquiry to be right-handed and to be French native speakers.

\subsubsection{Stimuli}

As target stimuli we used 38 words referring to perceptually identifiable parts of the human body. The relative vertical position of the body parts was determined by projecting a grid over a schematic picture of an upright standing human body (see Fig. 1A). Accordingly, each word was assigned a value between 1 and 10, depending on the vertical position of the body part that the word referred to (i.e. $1=$ hair, $10=$ toes). For the experiment we constructed word pairs referring to body parts varying in relative distance. To this end we used an automated procedure, whereby two words were randomly selected. Based on the relative distance between the body parts that the words referred to, the word pair was assigned to one of 9 different categories ( $1=$ close, $9=$ far $)$. This procedure was repeated for each participant until 20 word pairs were assigned to each of the 9 different categories. The rationale for creating different stimulus pairs for different participants was that the number of possible combinations of different body parts yielding different categories was bigger than the number of stimulus presentations per subject. By randomly creating stimulus pairs for different subjects we ensured that no systematic bias was introduced at the level of the stimuli. Each word pair was once presented in a congruent spatial position (e.g. eye above mouth: EYE/MOUTH) and once in an incongruent spatial position (MOUTH/EYE). Thus in total as target stimuli we used 360 word pairs referring to body parts according to a: 2 (spatial position: congruent vs. incongruent $) \times 9$ (distance: $1=$ close, $9=$ far $) \times 20$ repetitions design. In addition we included 38 filler words referring to animals that were matched for frequency and word length with the words referring to body parts (New, Pallier, Brysbaert, \& Ferrand, 2004).

In total we presented 840 trials: 420 trials (50\%) in which both words were from the same semantic category and 420 trials (50\%) in which the words were from a different semantic category. The 420 trials, in which both words were from the same category, consisted of 360 word pairs (42.9\%) referring to body parts and 60 word pairs referring to animals (7.1\%). The 420 trials in which the words were from a different semantic category consisted of 210 trials (25\%) in which the upper word referred to an animal and the lower word to a body part and 210 trials (25\%) in which the lower word referred to an animal and the upper word to a body part. Please note that with this design the relevant task dimension (semantic relatedness) was equally balanced in terms of the number of yes and no responses, while subjects always made the same response to target stimuli (word pairs referring to body parts) in a congruent and incongruent position.

\subsubsection{Design and procedure}

In the first experiment participants made a semantic relatedness judgment. Participants were instructed to indicate whether two simultaneously presented words were from the same category (body parts or animals) or not. Subjects were told that the words would always refer to either a body part and / or an animal. If the words were from the same category ( $50 \%$ of all trials), half of all participants were instructed to press the left button, but if the words were from a different category ( $50 \%$ of all trials), participants had to press the right button. The other half of all participants received opposite instructions. Subjects always responded with their right hand.

Each trial started with a fixation cross for 1000 to $2000 \mathrm{~ms}$, after which the word pair appeared centrally on the screen to which the subject responded. If the subject did not respond within $3000 \mathrm{~ms}$ after stimulus onset the next trial was initiated. A short error message was displayed if an incorrect response was made. Subjects performed 16 practice trials to familiarize with the task. During the experiment stimuli were presented in small blocks of 60 trials and no feedback was given about the average performance of the subject. In total the experiment took about $35 \mathrm{~min}$.

The analysis focused on target trials in which both words referred to body parts. For the analysis, trials with incorrect responses and trials that exceeded the subject's mean by more than two standard deviations were excluded from analysis. Reaction times and error rates to target trials were analyzed using a $2 \times 9$ repeated measures ANOVA with the factors Congruency (congruent vs. incongruent spatial position) and Distance $(1=$ close, $9=$ far $)$.

\subsection{Results}

On average subjects made incorrect categorizations in $9.4 \%$ of all trials, subjects missed responses in $1.6 \%$ of all trials and less than $1 \%$ of all trials were removed because they were more than 2 standard deviations above or below the subject's average reaction time. 
A

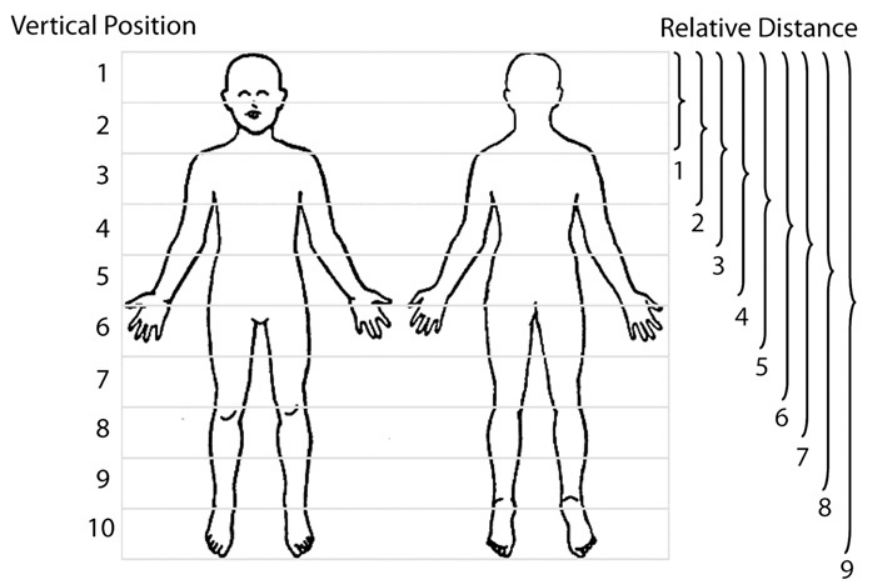

B

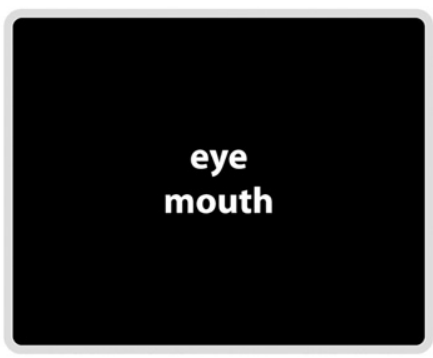

Congruent Spatial Position

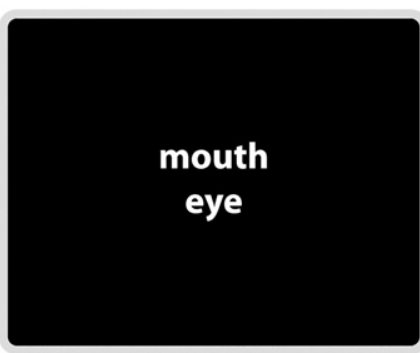

Incongruent Spatial Position

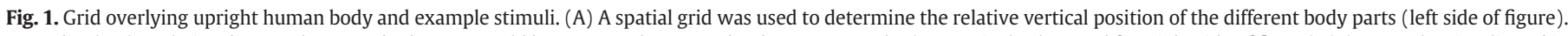

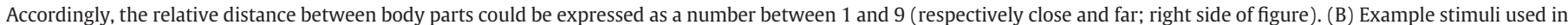
the experiment representing word pairs presented in a congruent spatial position (left side) or an incongruent spatial position (right side).

Comparison of target and filler trials showed that subjects responded faster to target trials representing two body parts ( $992 \mathrm{~ms}, \mathrm{SE}=33.6$ ) compared to filler trials representing a body part and an animal (1078 ms, SE = 37), t(20)<-12.7, $\mathrm{p}<.001$, filler trials representing an animal and a body part (1078 ms, SE $=42), \mathrm{t}(20)<-8.7, \mathrm{p}<.001$ and filler trials representing two animals $(1104 \mathrm{~ms}, \mathrm{SE}=31.9), \mathrm{t}(18)<-5.1$, $\mathrm{p}<.001$. The faster responses to target trials representing two body parts is likely related to the fact that this stimulus category was presented more often (42.9\% of all trials) than the other categories, thereby resulting in a facilitated recognition. In addition, it should be noted that in French many animal words are highly similar to words referring to body parts (e.g. cheval [horse] vs cheville [ankle]; pou [louse] vs. pouce [thumb]), thereby making word pairs referring to both an animal and a body part possibly more difficult to classify.

Error rates for target trials are represented on the left side of Fig. 2. No significant effect of Congruency was observed, $\mathrm{F}<1.0$. A marginally significant effect of Distance, $F(8,160)=1.7, p=.07, \eta^{2}=.09$, reflected a trend towards higher error rates with increased distance.

Reaction times for target trials are represented on the right side of Fig. 2. No main effect of congruency was found, $\mathrm{F}<1.0$. A significant

\section{Experiment 1}
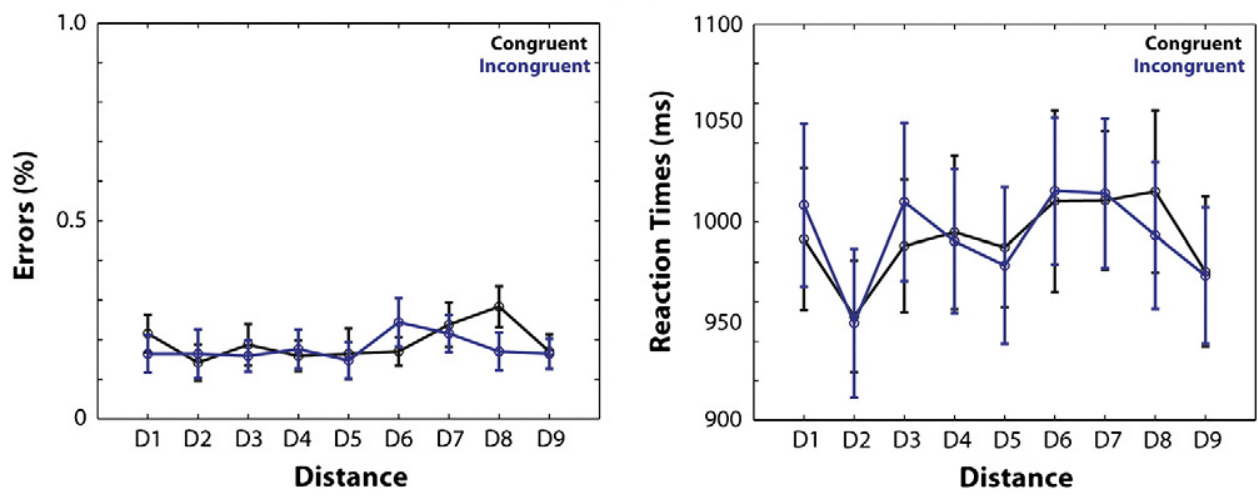

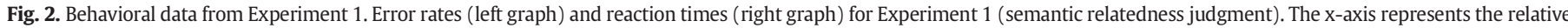

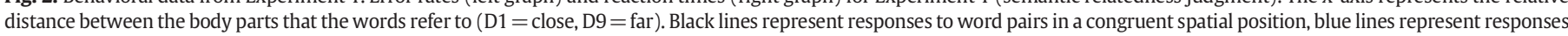
to word pairs in an incongruent spatial position. 
main effect of Distance was found, $\mathrm{F}(8,160)=2.9, \mathrm{p}<.005, \eta^{2}=.128$, reflecting overall slower reaction times with increased distance. A test of within-subjects contrasts revealed that the effect of distance was best accounted for by a cubic contrast, $\mathrm{F}(1,20)=12.4, \mathrm{p}<.005, \eta^{2}=.38$.

\subsubsection{Control for semantic category effects}

The first experiment showed a distance effect, reflected in slower reaction times and increased error rates with increased distance. The category with the largest distance difference (D9; see Fig. 1) always contained body parts at the most upper and lower part of the body. This category could therefore be considered a semantic category of the full human body (e.g. 'from head to toe'). To control for the possible confound that the distance effects were partly driven by this semantic category, we conducted an additional analysis, in which this category was excluded from analysis. Without category D9, still a strong effect of Distance was observed in the reaction times, $\mathrm{F}(7,140)=2.9, \mathrm{p}<.01$, and a marginally significant effect of Distance in the error rates, $F(7,140)=$ $2.0, p=.055$. These findings suggest that the distance effect is not driven by the semantic category representing the most upper and lower part of the human body.

\subsection{Discussion}

In the first experiment subjects made a semantic relatedness judgment to word pairs referring to body parts, to animals or to both. The spatial congruency and distance of the words referring to body parts were implicitly manipulated, by including word pairs in a congruent (EYE/MOUTH) or incongruent position (MOUTH/EYE) and word pairs referring to body parts that are close (e.g. EYE/MOUTH) or far in space (e.g. EYE/FOOT). In contrast to previous experiments using a similar design with words referring to real-world objects (Zwaan \& Yaxley, 2003b), no congruency effect was observed for words referring to body parts, presented in a congruent compared to an incongruent spatial position. Interestingly, though, an effect of distance was observed reflected in a slight increase in error rates and reaction times with increased distance between the body parts. This pattern of results is consistent with the mental scanning hypothesis, according to which scanning mental images across further distances takes longer than across shorter distances (Kosslyn et al., 1978). Therefore, the finding of a distance effect when subjects performed a semantic categorization of words referring to body parts could reflect that subjects to some extent activated a visual representation of the human body, specifying the relative distance between the respective body parts.

At least two mutually compatible explanations could underlie our failure to find a clear spatial congruency effect for words referring to body parts. First, it could be that body parts comprise a special semantic category that differs from ordinary objects in terms of visual associations. That is, many body parts can be perceived from both a 1st person and a 3 rd person perspective (e.g. legs and arms) and the spatial congruency of body parts differs strongly with the perspective taken (e.g. while lying on the beach in an armchair we see our own feet in the upper visual field instead of the lower visual field). In addition, some body parts are more difficult to perceive (e.g. buttocks, heel) than others (e.g. hand, or arm). Finally, in contrast to objects, such as cars or bottles that are sometimes occluded but can potentially be seen by moving around the object, this is not always possible with body parts. As a consequence, it could be that the visuo-spatial properties are less clearly defined for body parts than for objects.

In addition, it could be that spatial effects during language processing depend on the nature of the task and that spatial congruency effects are mainly elicited when subjects explicitly make a judgment about visual properties of the words' referents (Louwerse \& Jeuniaux, 2008, 2010). Several studies have shown that spatial effects during language processing are strongly modulated by top-down influences, such as perceptual or motor imagery (Hoenig, Sim, Bochev, Herrnberger, \& Kiefer, 2008; Louwerse \& Jeuniaux, 2008, 2010; Rueschemeyer, Lindemann, van Elk, \& Bekkering, 2009). Accordingly, it could be that a spatial congruency effect for body parts is only observed when the spatial dimension is made task-relevant and if it is clear to the subject what type of spatial body representation is relevant (i.e. observing a human body from a 3rd person perspective).

In sum, the absence of a spatial congruency effect in the first experiment could be related to the fact the spatial relation between body parts was not clearly defined (i.e. it was unclear whether spatial congruency was defined with respect to a 1st or 3rd person perspective) and to the fact that the spatial dimension was not task-relevant. To control for these possible confounds, we conducted a second experiment in which subjects were presented with the same stimuli as in the first experiment. In the second experiment subjects were explicitly instructed to make an iconicity judgment about the word pairs, deciding whether the spatial position of the words on the screen was congruent or incongruent with respect to the words' referents as imagined on an upright human body viewed from a 3rd person perspective.

\section{Experiment 2: Iconicity judgment}

\subsection{Participants}

In the second experiment 15 subjects participated ( 3 females, mean age $=22.7$ years), who received a financial remuneration for participation and who had not participated in the previous experiment. Subjects declared themselves through informal verbal inquiry to be right-handed and to be French native speakers.

\subsubsection{Design and procedure}

As stimuli we used the same 38 words referring to body parts as in the previous experiment. In total the experiment consisted of 360 word pairs referring to body parts according to a: 2 (spatial position: congruent vs. incongruent) $\times 9$ (distance: $1=$ close, $9=$ far $) \times 20$ repetitions design.

In the second experiment subjects were asked to make iconicity judgments. At the beginning of the experiment participants were instructed to indicate if the spatial position of the words on the screen was congruent or incongruent with respect to the actual spatial position of the body parts to which the words referred. Participants were instructed to judge the spatial arrangement with respect to an upright human body as viewed from a 3rd person perspective. If the spatial position of the words was congruent subjects were required to press the left button of a serial response box and if the spatial position was incongruent subjects were required to press the right button. Subject always responded with their right hand. At the beginning of the experiment subjects performed 16 practice trials to familiarize with the task.

For the analysis, trials with incorrect responses and trials that exceeded the subject's mean by more than two standard deviations were excluded from analysis. Reaction times and error rates to target trials were analyzed using a $2 \times 9$ repeated measures ANOVA with the factors Congruency (congruent vs. incongruent spatial position) and Distance $(1=$ close, $9=$ far $)$.

\subsection{Results}

On average subjects made incorrect responses in $9.8 \%$ of all trials, subjects missed responses in $2.3 \%$ of all trials and $0.2 \%$ of all trials was removed because they were more than 2 standard deviations above or below the subjects average reaction time.

Error rates are represented on the left side of Fig. 3. Analysis of the error rates revealed a main effect of Congruency, $F(1,14)=11.7$, $\mathrm{p}<.005, \eta^{2}=.46$, reflecting more errors for words in an incongruent 
compared to a congruent spatial position. A main effect of Distance, $\mathrm{F}(8,112)=38.5, \mathrm{p}<.001, \eta^{2}=.73$, reflected that subjects made more errors to words referring to body parts that are close together compared to body parts that are further apart. An interaction between Congruency and Distance, $\mathrm{F}(8,112)=2.1, \mathrm{p}<.05, \eta^{2}=.13$, reflected that the effect of distance was slightly stronger for words presented in an incongruent compared to a congruent spatial position. Analysis of the contrasts revealed that the main effect of Distance in error rates was best explained, by assuming a 5th order contrast, $\mathrm{F}(1,14)=6.5, \mathrm{p}<.05$.

Reaction times are represented on the right side of Fig. 3. A main effect of Congruency, $F(1,14)=33.8, \eta^{2}=.71, \mathrm{p}<.001$, reflected slower reaction times to words presented in an incongruent compared to a congruent spatial position. A main effect of Distance, $F(8,112)=20.2$, $\eta^{2}=.59, \mathrm{p}<.001$, reflected slower reaction times to words referring to body parts that are close together compared to body parts that are further apart. No significant interaction was found $(\mathrm{F}<1)$. An analysis of the contrasts revealed that the main effect of Distance was best explained by assuming a quadratic contrast, $\mathrm{F}(1,14)=6.5, \mathrm{p}<.05$.

\subsubsection{Control for repetition effects}

In the second experiment body parts were randomly combined to generate word pairs that differed in the spatial proximity of the words' referents. However, by definition the possible number of word combinations for body parts that are further apart (e.g. relative distance D8 or D9) is more limited than the possible number of word combinations for body parts that are close in space (e.g. relative distance D1 or D2). As a consequence, words referring to the most upper or lower parts of the body may have been selected more often and the decrease in reaction times for words referring to body parts that are further apart may actually reflect repetition priming or recency effects for frequently presented words (Segui \& Grainger, 1990). To control for this possible confound we conducted an additional analysis in which we split the data in three different blocks. If the body distance effect could be attributed to stimulus repetitions, a stronger body distance effect should be expected in the final compared to the first block (i.e. interaction between Block and Distance). The additional analysis showed a main effect of Block, $F(2,28)=10.0, p<.001, \eta^{2}=.42$, reflecting an overall decrease in reaction times with increased practice. However, Block did not interact with Distance $(F<1)$, suggesting that the body distance effect was constant across blocks.

\subsubsection{Control for semantic category effects}

Similar to the first experiment, we conducted an additional analysis to control for the possible confound that the distance effects were partly driven by the semantic category representing the most upper and lower part of the human body. Without category D9, still a strong effect of Distance was observed in the reaction times, $F(7,98)=21.3$, $\mathrm{p}<.001$, and in the error rates, $\mathrm{F}(7,98)=38.9, \mathrm{p}<.001$. These findings rule out the possible confound that the distance effect is driven by the semantic category representing the most upper and lower part of the body.

\subsubsection{Individual subjects analysis}

In the second experiment we tested a relatively low number of subjects $(n=15)$, especially compared to previous studies on this topic (e.g. Zwaan \& Yaxley, 2003b). To assess the robustness of the spatial congruency effect and the body distance effect, we conducted an additional analysis. For each subject we calculated the congruency effect by subtracting the average reaction times to congruent from incongruent trials. In addition, for each subject we calculated the regression coefficient that best fitted the data for the body distance effect (e.g. a negative regression coefficient is indicative of faster reaction times with increased distance between body parts). It was found that 14 out of 15 subjects showed a spatial congruency effect and for 14 out of 15 subjects the data was fitted with a negative regression coefficient, indicative of a body distance effect at an individual subject level. Together these findings further support the robustness of the spatial congruency effect and the distance effect in the second experiment.

\subsection{Discussion}

In the second experiment subjects made iconicity judgments to word pairs referring to body parts. First, a congruency effect was observed, reflected in faster reaction times to word pairs that were presented in a spatially congruent compared to an incongruent position. The congruency effect likely reflects that a spatial match between the body representation and the visual presentation of the words on the screen results in faster reaction times. That is, because body parts have a predefined spatial relation, iconic body word pairs (i.e. corresponding to the prototypical arrangement of body parts) are processed faster than reverse-iconic body word pairs. When making a semantic categorization judgment (Experiment 1) no spatial congruency effect was observed but subjects still activated information about the relative distance between body parts, as evidenced by a distance effect. In contrast, the finding of a spatial congruency effect in the second experiment suggests that subjects activated spatial body representations in an absolute frame of reference (i.e. a body as viewed from a 3rd person perspective) when making iconicity judgments.

\section{Experiment 2}
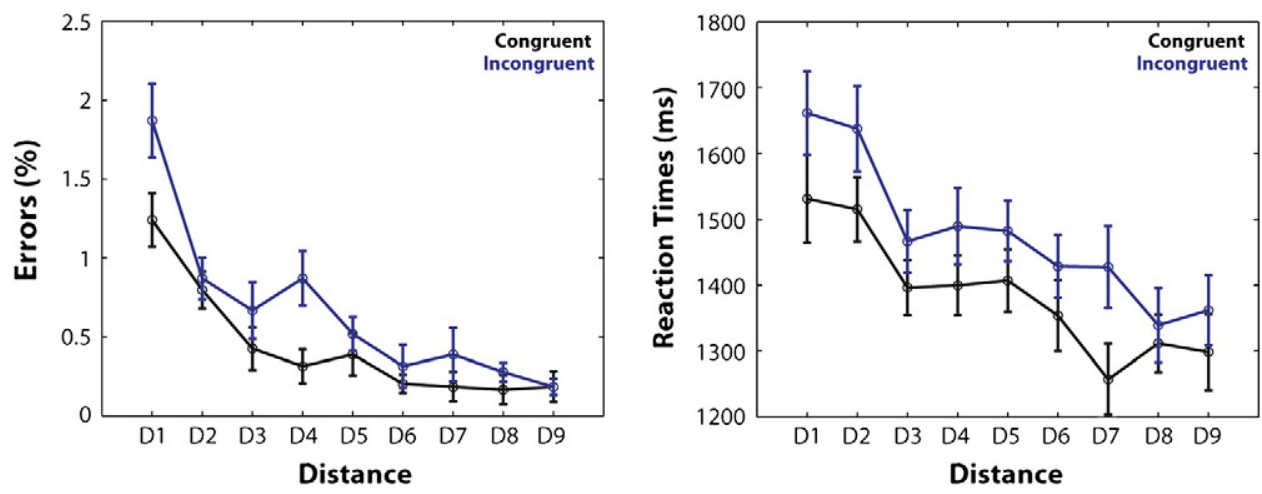

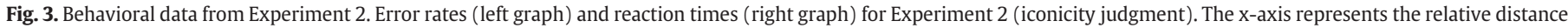

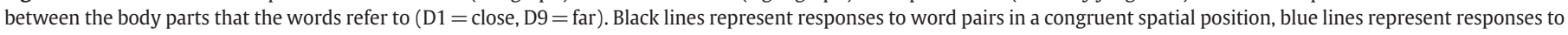
word pairs in an incongruent spatial position. 
Second, a distance effect was observed, which-in contrast to Experiment 1 -was reflected in a decrease in reaction times and error rates for body parts that were spatially further apart. The distance effect as observed in the second experiment can be considered as a specific instance of the symbolic distance effect, that is typically reflected in faster reaction times in a size estimation task if the difference between the sizes increases (Moyer \& Bayer, 1976; Moyer \& Landauer, 1967). Previous studies have shown distance effects for the comparison of animals (Paivio, 1975), geographical locations (Maki, 1981) and familiar objects (Dean et al., 2005; Marschark \& Paivio, 1981). Although recent studies have reported a symbolic distance effect in a task requiring subjects to make explicit comparisons between the relative distances of body parts (Smeets et al., 2009; Struiksma et al., 2011), in the present study, a symbolic distance effect was observed even though the relative distance between body parts was irrelevant to the subject's task. In addition, previous work established the distance effect for the processing of body parts only at a relatively coarse level. As a consequence it could partly reflect a categorical organization of body parts into hierarchically organized clusters (Andersen, 1978; Blasing, Schack, \& Brugger, 2010). Here, we report that the symbolic distance effect for body parts was found for body parts that were both close and far in space.

An important question is why the semantic categorization task resulted in a distance effect that is compatible with the mental scanning hypothesis (i.e. longer RTs with increased distance), whereas the iconicity judgment task resulted in a distance effect that is compatible with the symbolic distance effect (i.e. shorter RTs with increased distance). One important reason for why the iconicity judgment task resulted in a decrease in reaction times with increased distance on the body surface is the following. When making an iconicity judgment, subjects were required to indicate if the observed spatial position of words on a screen was congruent with their memorized location of the words' referents. In other words: the iconicity judgment required a comparison between an observed spatial arrangement and a memorized spatial arrangement of body parts. This comparison process was facilitated if the spatial distance between the words' referents was larger, because comparing large distance differences is easier than comparing small distance differences (Moyer \& Bayer, 1976; Moyer \& Landauer, 1967). On this account, it is the actual comparison process between an observed and a memorized spatial representation that results in slower reaction times for body parts that are close in space during the iconicity judgment task. In contrast, during a semantic categorization task no comparison is required and the reading of the words on the screen likely triggers the activation of a memorized spatial representation. In case the distance between both body parts increases it is more difficult to determine whether both words belong to the same semantic category (i.e. scanning the mental image of the human body takes longer) and this is reflected in slower reaction times.

Together, the findings from the second experiment suggest that making a decision about the visual properties of body semantics is accompanied by the activation of a detailed spatial body representation, specifying both the location and the relative distance of body parts. An interesting question is what possible neural mechanism could underlie the spatial congruency effect and the symbolic distance effect observed in the second experiment. Previous studies have associated impaired knowledge of body semantics with left hemispheric lesions (Coslett et al., 2002; Kemmerer \& Tranel, 2008; Laiacona et al., 2006; Schwoebel \& Coslett, 2005). However, it is unclear whether these patients were primarily impaired at relating the names of body parts to their corresponding location or suffered from a general deficit in their body schema. Spatial representations of the human body have been described at many different levels of the brain, such as the primary motor cortex and somatosensory cortex (Flanders, 2005), but also in the extrastriate body area (Chan, Kravitz, Truong, Arizpe, \& Baker, 2010; Orlov, Makin, \& Zohary, 2010). A common characteristic feature of these areas is that the body is represented in a somatotopically organized fashion in both hemispheres, although the abovementioned clinical studies suggested a left hemispheric dominance of body semantics. Finally, several studies have shown that both hemispheres have specialized linguistic functions. For instance, whereas the left hemisphere is mainly involved in retrieving literal word meaning, the right hemisphere may support the retrieval of more remote semantic associations, as for instance in processing metaphors or discourse (Beeman, Bowden, \& Gernsbacher, 2000; Schmidt, DeBuse, \& Seger, 2007). Thus, at present it is unclear whether a clear hemispheric specialization exists, supporting the retrieval of spatial information associated with body semantics.

To investigate the possibility of hemispheric specialization for body semantics, in a third experiment we used the same paradigm as in the second experiment, but rather than presenting the words centrally on the screen, word pairs were presented to the left and right visual field (cf. Zwaan \& Yaxley, 2003a). If body semantics are selectively represented in the left hemisphere (Coslett et al., 2002; Kemmerer \& Tranel, 2008; Laiacona et al., 2006; Schwoebel \& Coslett, 2005), a stronger spatial congruency effect and symbolic distance effect should be expected for words presented to the right visual field compared to the left visual field. Alternatively, if body semantics recruits spatial processing in both hemispheres, a comparable spatial congruency effect and symbolic distance effect may be expected for words presented in both visual fields.

\section{Experiment 3: Iconicity judgments in the left and right visual field}

\subsection{Methods}

\subsubsection{Participants}

In the third experiment 14 right-handed and French speaking participants were tested ( 3 females, mean age $=21.2$ years) who did not participate in the previous experiments. Data from 2 participants was excluded from analysis because of errors in more than $25 \%$ of all trials.

\subsubsection{Stimuli}

The same stimuli were used as in the second experiment. For each category, each word pair was presented both in the left visual field and in the right visual field. Thus in total the experiment consisted of 720 trials, according to the following design: 2 (Visual field: left vs. right $) \times 2$ (Spatial position: congruent vs. incongruent $) \times 9$ (Distance: $1=$ close, $9=$ far $) \times 20$ repetitions. Word pairs were presented 100 pixels (approximately 1.8 visual degrees) to the left or right side of the centre of the screen. At this distance it was still possible to read the words while maintaining fixation at the fixation cross. Previous studies on lateralization of word processing have used comparable settings for visual word presentation (see for instance: Graves, 1983; Regard, Landis, \& Graves, 1985).

\subsubsection{Design and procedure}

The experimental design and procedure were similar as in Experiment 2 . In addition, subjects were explicitly instructed to maintain fixation on the central fixation cross and to avoid making eye movements to the word pairs. Compliance with this instruction was checked by having subjects perform two subsequent practice sessions, in which subjects familiarized with the task. In addition, during the experiment subject's eye movements were measured with a webcam, aligned with the experimental events and stored for offline analysis. Visual inspection of these videos confirmed that the remaining 12 participants managed to maintain fixation throughout the experiment. In contrast to the first and second experiment, subjects had $5000 \mathrm{~ms}$ after stimulus onset to respond before the word pair was replaced with a fixation cross. Due to the increased task difficulty, a shorter response interval would have resulted in too many missed 
responses. Similar to the previous experiments, subjects always responded with their right hand, by pressing the left key of a response box if the body parts were in a congruent spatial position and the right key if the body parts were in an incongruent position. Reaction times and error rates were analyzed using a $2 \times 2 \times 9$ repeated measures ANOVA with the factors Visual field (left vs. right), Congruency (congruent vs. incongruent spatial position) and Distance ( $1=$ close, $9=$ far $)$

\subsection{Results}

Incorrect responses were detected in $10.6 \%$ of all trials, missed responses in $0.9 \%$ of all trials and less than $0.2 \%$ of all trials was removed because it was more than 2 standard deviations above or below the subjects average reaction time.

Error rates are represented in the left side of Fig. 4. For the analysis of the error rates a main effect of Congruency was found, $\mathrm{F}(1,11)=6.5$, $\eta^{2}=.37, \mathrm{p}<.05$, reflecting more errors for words in an incongruent compared to a congruent spatial position. A main effect of Distance, $\mathrm{F}(8,88)=56.0, \eta^{2}=.84, \mathrm{p}<.001$, reflected that subjects made more errors to words referring to body parts that are close together compared to body parts that are further apart. A marginally significant interaction between Congruency and Distance, $F(8,88)=1.4$, $\eta^{2}=.15, \mathrm{p}=.06$, reflected that the effect of distance was slightly stronger for words presented in an incongruent compared to a congruent spatial position. No other significant interactions were observed. Finally, a main effect of Visual field was found, $F(1,11)=$ $10.5, \eta^{2}=.49, \mathrm{p}<.01$, reflecting more errors for words presented in the left visual field compared to the right visual field. Analysis of the contrasts revealed that the main effect of Distance was best explained, by assuming a cubic contrast, $\mathrm{F}(1,11)=43.6, \mathrm{p}<.001$.
Reaction times are represented on the right side of Fig. 4. A main effect of Congruency, $\mathrm{F}(1,11)=9.4, \mathrm{p}<.05, \eta^{2}=.46$, reflected slower reaction times to words presented in an incongruent compared to a congruent spatial position. A main effect of Distance, $F(8,88)=37.4$, $\mathrm{p}<.001, \eta^{2}=.77$, reflected slower reaction times to words referring to body parts that are close together compared to body parts that are further apart. No significant interactions were found $(\mathrm{F}<1)$. Finally, a main effect of Visual field, $F(1,11)=17.1, p<.005, \eta^{2}=.61$, reflected slower reaction times to words presented in the left visual field $(1801 \mathrm{~ms}$, $\mathrm{SE}=100)$ compared to the right visual field $(1736 \mathrm{~ms}, \mathrm{SE}=99)$. An analysis of the contrasts revealed that the main effect of distance was best explained by a quadratic contrast, $\mathrm{F}(1,11)=9.2, \mathrm{p}<.05$.

\subsubsection{Control for repetition effects}

In an additional analysis we again controlled for the possible confound that the body distance effect could be attributed to differences in frequency of presentation between the different stimulus categories. To this end, the data was split in 3 different blocks (data from 1 subject was not included in the additional analysis, due to insufficient data points). A significant main effect of Block, $F(2,20)=33.8$, $\mathrm{p}<.001, \eta^{2}=.77$, reflected an overall decrease in reaction times with increased practice. Importantly, Block did not interact with Distance $\left(F(16,160)=1.4, p=.14, \eta^{2}=.12\right)$, suggesting that the body distance effect did not differ across the different blocks.

\subsubsection{Control for semantic category effects}

Similar to the first and second experiment, we conducted an additional analysis to control for the possible confound that the distance effects were partly driven by the semantic category representing the most upper and lower part of the human body. Without category D9, still a strong effect of Distance was observed in the reaction times, $\mathrm{F}(7,77)=34.0, \mathrm{p}<.001$, and in the error rates, $\mathrm{F}(7,77)=59.4$,

\section{Experiment 3}
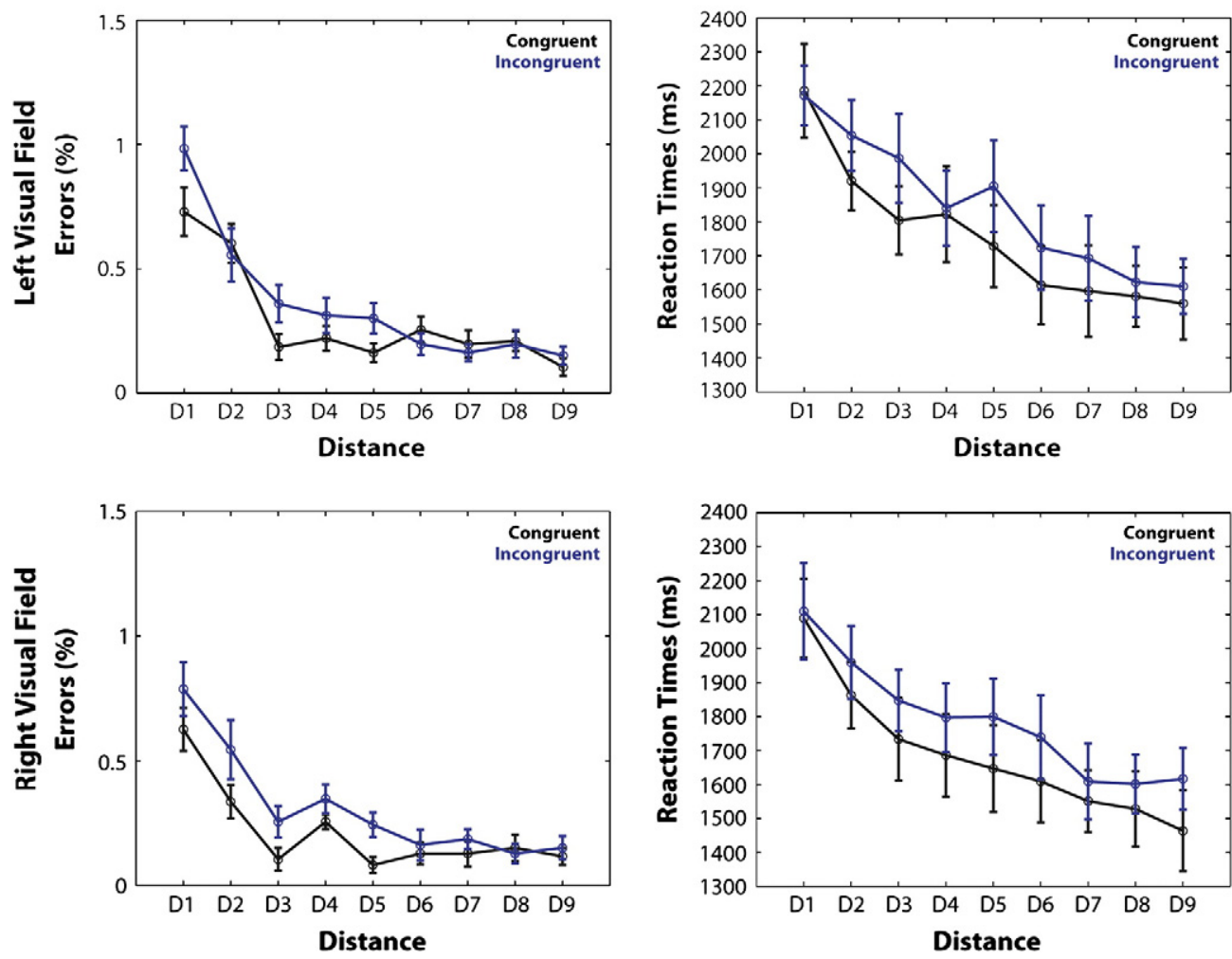

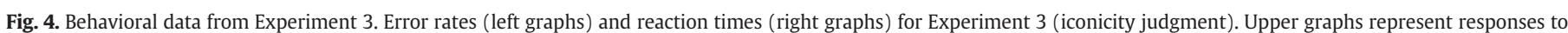

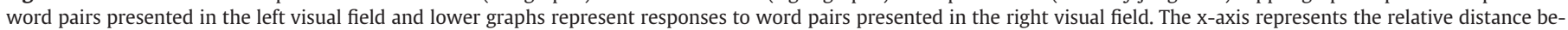

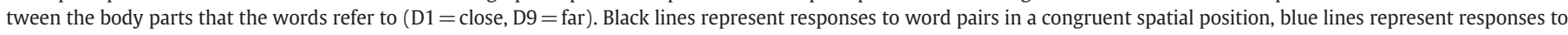
word pairs in an incongruent spatial position. 
$\mathrm{p}<.001$. These findings rule out the possible confound that the distance effect is driven by the semantic category representing the most upper and lower part of the body.

\subsubsection{Individual subjects analysis}

In an additional analysis, we assessed the robustness of the spatial congruency effect at an individual subject level. It was found that 11 out of 12 subjects showed the spatial congruency effect and the body distance effect was observed in all participants (as evidenced by negative regression coefficients for all subjects), thereby confirming the robustness of the effects observed, despite the relatively low number of subjects tested.

\subsection{Discussion}

The third experiment replicates findings from the second experiment, by showing a congruency effect for body parts presented in a congruent compared to an incongruent spatial position, and a distance effect, reflected in faster reaction times to body parts that are further apart. However, neither the congruency effect nor the distance effect was modulated by the visual field to which the word pairs were presented. This finding suggests that the spatial congruency effect for body parts and the distance effect are most likely mediated by activating spatial information regarding the human body across both hemispheres. It could be that patients with impaired knowledge of body semantics and damage to left temporal areas (Coslett et al., 2002; Laiacona et al., 2006; Schwoebel \& Coslett, 2005) are specifically impaired in accessing body semantics at a lexico-semantic level (i.e. relating the names of words to the corresponding body parts), whereas they have a spared spatial representation of the human body. The possible functional and neural implications of these findings will be discussed in more detail in the general discussion.

\section{General discussion}

In three behavioral experiments we assessed the functional organization of body semantics with respect to congruency, distance on the body surface, and stimulated hemifield. Two main findings support the view that the processing of body semantics is accompanied by the activation of a detailed spatial body representation. First, when making a semantic relatedness judgment (Experiment 1 ) a distance effect was observed, reflected in slower responses to body parts that are further apart. In contrast, when making an iconicity judgment (Experiments 2 and 3) a distance effect was observed that was reflected in relatively slow responses to body parts that are close in space (e.g. EYE / MOUTH) and faster responses to body parts that are far in space (e.g. EYE / FOOT). Second, when asked to make iconicity judgments about word pairs referring to body parts (Experiments 2 and 3 ) but not during semantic relatedness judgments (Experiment 1), participants were faster if the body parts were in a congruent spatial position (EYE / MOUTH) compared to an incongruent spatial position (MOUTH / EYE). We will first discuss effects of spatial congruency and next focus on the possible functional and neural mechanisms underlying the distance effects.

\subsection{Spatial congruency effect}

At a functional level the congruency effect likely reflects a match or mismatch between a spatial representation of the human body, activated by reading the words, and the location of the words on the screen. That is, to make an iconicity judgment of word pairs one needs to access a representation of the human body, specifying the relative spatial position of body parts. If the activated representation matches with the actual spatial position of the words, this results in a facilitation of response times, whereas a mismatch results in delayed response times. A similar mechanism is likely involved in the spatial Stroop effect, in which the task-irrelevant location of a stimulus is automatically encoded and affects word recognition (Lu \& Proctor, 2001). In contrast to the spatial Stroop effect, in the present study a spatial congruency effect was only observed when the spatial position of the words was relevant to the subject's task (Experiments 2 and 3), whereas no interference was observed if the spatial position was irrelevant (Experiment 1). This finding suggests that the present congruency effect is not driven purely by bottom-up stimulus features, but rather by the requirement to compare observed with memorized spatial locations of body parts.

The fact that the spatial congruency effect was only found during an iconicity judgment task but not during a semantic categorization task seems to be in apparent conflict with the classical finding of a congruency effect when making a semantic relatedness judgment about words referring to objects (Zwaan \& Yaxley, 2003b). At least two factors could underlie this apparent discrepancy. First, the relation between words and their visual associations is more complicated in the case of body parts than for external objects. We perceive human bodies both from a first and a 3rd person perspective and in addition not all body parts can be easily perceived or are perceived equally often. An interesting possibility for future research would be to make a direct comparison between body semantics and object semantics (e.g. have car experts judge the spatial congruency of word pairs referring to car parts), to investigate the possibility that the automaticity of visuo-spatial representations during language processing differs between semantic categories.

Second, only in the case of an iconicity judgment it was clear to the subject what specific visual body representation needed to be activated in order to solve the task. The finding of a spatial congruency effect during the iconicity judgment task suggests that subjects actually accessed a spatial representation of the human body that specified the absolute position of body parts. The spatial congruency effect was probably related to the instruction to judge the spatial arrangement of the words "with respect to an upright human body as viewed from a 3rd person perspective'. This instruction likely resulted in the grounding of the spatial body representation in an absolute rather than a relative frame of reference. In contrast, in the first experiment when the frame of reference was left implicit no spatial congruency effect was observed, whereas information about the relative position of body parts was preserved as evidenced by a distance effect. The finding of a spatial congruency effect for words referring to body parts can be considered an extension of previous studies, showing a visual processing advantage for bodies that are aligned with the observer's body axis (Lobmaier \& Mast, 2007; Lopez et al., 2009; Reed et al., 2003). An interesting possibility would be to investigate if manipulating one's body posture or body position (e.g. having subjects perform the task while lying or while in an upside-down position) has similar effects on the processing of body semantics, as it has on the processing of visual body representations.

\subsection{Distance effects}

In addition to the spatial congruency effect, in all experiments a distance effect was observed, reflected in a modulation of reaction times with increased distance. The distance effect provides further support for the notion that subjects implicitly retrieved detailed information about the relative position of body parts when processing body semantics. When making a semantic categorization (Experiment 1 ) reaction times increased with increased distance, whereas when making an iconicity judgment (Experiments 2 and 3 ) reaction times decreased with increased distance between the body parts. The finding of an increase in reaction times with increased perceptual distance between the words' referents (Experiment 1 ) is reminiscent of visual imagery findings, in which the time required to scan mental images increases with longer distances (Kosslyn et al., 1978). Similarly, in a language experiment it was found that participants were faster in a part verification task when responding to object parts that were close compared to far with respect to the perspective imposed by the preceding sentence 
(Borghi et al., 2004). In the present study, in case the distance between both body parts increased it was probably more difficult to determine whether both words belonged to the same semantic category (i.e. scanning the mental image of the human body takes longer) than when the referring body parts were close in space.

In contrast, when making an iconicity judgment reaction times decreased with increased distance between the body parts (Experiments 2 and 3), which can be considered an instance of the symbolic distance effect (Moyer \& Bayer, 1976; Moyer \& Landauer, 1967). When making an iconicity judgment, subjects were required to indicate if the observed spatial position of words on a screen was congruent with their memorized location of the words' referents. In other words: the iconicity judgment required a comparison between an observed spatial arrangement and a memorized spatial arrangement of body parts. This comparison process was facilitated if the spatial distance between the words' referents was larger, because comparing large distance differences is easier than comparing small distance differences (Moyer \& Bayer, 1976; Moyer \& Landauer, 1967).

A plausible explanation of the symbolic distance effect, is provided by the place coding model (for review, see: Verguts, Fias, \& Stevens, 2005). This model assumes that the relevant stimuli, for instance numbers, dates, geographical locations and in our case: body parts, are somehow spatially represented. According to the place coding model reading for example the word 'nose' results, due to spreading activation related to the processing of a specific stimulus, in the activation of neighboring representations as well (e.g. eye, mouth, etc.). As a consequence, due to overlapping representations two stimuli that are nearby are more difficult to discern than two stimuli that elicit non-overlapping representations. This leads to interference that is reflected in slower responses to body parts that are close in space and faster responses to body parts that are spatially further apart. Please note that this explanation and the present data remain agonistic about the type of spatial representation that is involved at the neural level: the symbolic distance effect for body parts could be mediated by the spreading of activation in motor, somatosensory or even visual areas (e.g. the extrastriate body area; EBA) that follow a somatotopic representation of the human body. Future studies would need to address the precise relation between body semantics and the precise neural structures supporting spatial body representations.

The present findings on congruency and distance effects are in line with previous studies that have shown congruency effects for the processing of words referring to real-world objects (e.g. roof / attic; Zwaan \& Yaxley, 2003a, 2003b) and symbolic distance effects after verbal comparison of animals (Noordzij \& Postma, 2005; Paivio, 1975) and geographical locations (Maki, 1981). In addition the findings from Experiment 2 and 3 are in agreement with the findings from recent studies in which subjects were required to compare the distance between two body widths (Smeets et al., 2009; Struiksma et al., 2011). In these studies a decrease in reaction times was observed with increased distance difference in the horizontal plane and in addition a relation was observed between the distance effect and body shape concern. However, in previous studies subjects were explicitly required to make a distance comparison between body parts, whereas in the present study the relative distance between body parts was task-irrelevant. Still, the finding of a distance effect when making an iconicity judgment suggest that tasks like this may be usable for clinical purposes as well (e.g. assessment of body image and body schema disturbances).

The present study has important implications for theories on embodied cognition. Two key questions in discussions on embodied cognition are (1) whether activation in modality-specific brain areas is automatic and bottom-up or driven by contextual and top-down influences (Pulvermuller \& Fadiga, 2010) and (2) to what extent activation in modality-specific brain areas is necessary for language understanding (Fischer \& Zwaan, 2008; Mahon \& Caramazza, 2008). The finding that both the spatial congruency effect and the distance effect are modulated by task requirements (semantic categorization vs. iconicity judgment) argues against the view that the activation of spatial information is automatic and necessary for language understanding (see also: Hoenig et al., 2008; Louwerse \& Jeuniaux, 2008, 2010; Rueschemeyer et al., 2009). Only when participants made an iconic judgment about the spatial properties of body semantics a clear spatial congruency effect was observed. In addition, making a semantic categorization response resulted in a distance effect that is consistent with the mental scanning hypothesis, whereas making an iconicity judgment resulted in a distance effect that is consistent with the symbolic distance effect. Thereby, the present study suggests that different perceptual representations are activated depending on the task requirements. Our data suggests that a structural description of the human body specifying the absolute position of body parts (Schwoebel \& Coslett, 2005) is only activated when information about the absolute position of body parts as viewed from a 3rd person perspective is relevant to the subject's task. In contrast, when the spatial perspective was left implicit as in the semantic categorization task, only information about the relative position of body parts was activated, as evidenced by the distance effect. The selective activation of spatial information in association with body semantics can be considered an instance of Allport's selection for action principle (1987) according to which a subject's task intention determines the activation of relevant information.

\subsection{Limitations and open issues}

In addition to these main findings several minor issues require discussion. First, when selecting the word pairs referring to body parts we did not control for lexico-semantic features or frequency of presentation for the different body parts. This was an inevitable consequence of the fact that the number of words referring to body parts is limited and that the different body parts could be combined in many different ways to construe word pairs representing different distances between the words' referents. However, the finding that no congruency effect was observed in the first experiment, in which subjects made a semantic relatedness judgment, suggests that the effects in the other experiments cannot be attributed to differences in lexico-semantic features. In addition, the random assignment of body words to different body pairs ensured that over subjects the different possible combinations of body pairs were equally balanced out, which would not have been possible with a predefined assignment of body parts to different categories. Finally, in an additional analysis it was found that the distance effect was constant over experimental blocks, thereby further minimizing the possibility that the effect can be attributed to repeated stimulus presentations.

In the first experiment subjects always responded to congruent and incongruent word pairs by pressing the same button, whereas in Experiments 2 and 3 congruent and incongruent word pairs involved different button press responses. The fact that in Experiments 2 and 3 the congruency effects were measured by using different response buttons, was an inevitable consequence of using a spatial iconicity task. That is, in a spatial iconicity task subjects are explicitly required to classify stimuli according to their spatial congruity (i.e. congruent or incongruent), which always involves a selection between two responses. It is difficult to come up with a design in which this iconicity decision can be mapped to a single button press without introducing other confounds (e.g. a go / no go task would result in responses to congruent and incongruent trials being acquired in different blocks and thereby in possible blockorder effects). However, it is unlikely that the different distance and congruency effects observed between the experiments could be partly attributed to differences in the response selection process (i.e. selecting one vs. two buttons to respond). First, previous studies have indicated that spatial compatibility effects, such as the Simon effect, the Stroop effect, or the SNARC effect likely arise at the level of response selection, as stimuli and responses share overlapping features (i.e. responding to a stimulus at the left side with the left hand; Keus, Jenks, \& Schwarz, 
2005; Lu \& Proctor, 1995). In contrast, in the present study there was no overlap between the relevant stimulus dimension (congruent vs. incongruent spatial position) and the mapping of the response buttons (left vs. right), thereby making the possibility that the effects observed arise at the level of response selection less likely. Second, it should be noted that although responses to word pairs in a congruent and incongruent spatial position involved different button presses, the finding that the distance effect was constant for both congruent and incongruent responses suggests that this effect was not modulated by the selection of a response button. Thus the comparison of the distance effect between the different experiments seems warranted and the finding that the distance effect is modulated by the task (semantic relatedness judgment vs. iconicity judgment) suggests that the task requirements are the driving factor underlying differences between Experiments 1 and 2 rather than differences in response selection.

In the third experiment it was found that subjects responded slower and were less accurate to word pairs presented in the left compared to the right visual field, thereby replicating previous findings (Bryden, 1965; Graves, 1983; Hugdahl, 2000; Mishkin \& Forgays, 1952; Regard et al., 1985) and supporting the compliance of subjects with the instruction to maintain fixation. However, in the present study the right visual field advantage could also be partly confounded with the fact that participants always responded with their right hand, thereby resulting in an attentional bias to the right side (e.g. Eimer, Forster, Van Velzen, \& Prabhu, 2005) and/or an additional congruence between field of presentation and side of responding (Weems \& Zaidel, 2005). Neither the congruency effect nor the distance effect was modulated by the visual field of presentation. This result stands in apparent contrast to the findings by Zwaan and Yaxley (2003a, 2003b), who found a spatial congruency effect only for words presented to the left visual field in line with the proposed dominance of the right hemisphere for processing visuo-spatial relations. However, it should be pointed out that the present study investigated spatial relations for a different class of stimuli (i.e. body parts). The finding that the congruency effect and distance effect for body parts was not modulated by the visual field to which the stimuli were presented, is in line with the idea that the brain contains multiple bilateral representations of the human body (de Vignemont, 2010). Exactly which body representation underlies the effects observed in the present study remains to be determined in future research.

\section{Conclusions}

The main finding of the present study is that the processing of bodysemantics results in the activation of a detailed visuo-spatial body representation that is dependent on the specific task requirements. When making a semantic categorization response we found a distance effect that is consistent with a visual imagery explanation. In contrast, when making an iconicity judgment we found a distance effect that is consistent with the symbolic distance effect and in addition we observed a spatial congruency effect. These findings underline the flexibility of embodied language processing with respect to body semantics.

\section{Acknowledgments}

We would like to thank two anonymous reviewers for their helpful comments on a previous version on this manuscript. The present study was supported by the Marie Curie Intra European Fellowship within the Seventh European Community Framework Program (IEF grant 252713 to MVE).

\section{References}

Allport, A. (1987). Selection for action: Some behaviorial and neurophysiological considerations of attention and action. In H. Heuer, \& A. F. Sanders (Eds.), Perspectives on Perception and Action (pp. 395-419). Hillsdale, NJ, England: Lawrence Erlbaum Associates.
Andersen, E. (1978). Lexical universals of body-part terminology. In J. H. Greenberg (Ed.), Universals of human language. Word structure, 3. (pp. 335-368) Stanford, CA: Stanford University Press.

Barton, J. J. (2003). Disorders of face perception and recognition. Neurologic Clinics, 21, 521-548.

Beeman, M. J., Bowden, E. M., \& Gernsbacher, M. A. (2000). Right and left hemisphere cooperation for drawing predictive and coherence inferences during normal story comprehension. Brain and Language, 71, 310-336.

Blasing, B., Schack, T., \& Brugger, P. (2010). The functional architecture of the human body: Assessing body representation by sorting body parts and activities. Experimental Brain Research, 203, 119-129.

Borghi, A. M., \& Cimatti, F. (2010). Embodied cognition and beyond: Acting and sensing the body. Neuropsychologia, 48, 763-773.

Borghi, A. M., Glenberg, A. M., \& Kaschak, M. P. (2004). Putting words in perspective. Memory \& Cognition, 32, 863-873.

Borst, G., Kosslyn, S. M., \& Denis, M. (2006). Different cognitive processes in two imagescanning paradigms. Memory \& Cognition, 34, 475-490.

Bryden, M. P. (1965). Tachistoscopic recognition, handedness, and cerebral dominance. Neuropsychologia, 3, 1-8.

Buxbaum, L. J., \& Coslett, H. B. (2001). Specialised structural descriptions for human body parts: Evidence from autotopagnosia. Cognitive Neuropsychiatry, 18, 289-306.

Chan, A. W., Kravitz, D. J., Truong, S., Arizpe, J., \& Baker, C. I. (2010). Cortical representations of bodies and faces are strongest in commonly experienced configurations. Nature Neuroscience, 13, 417-418.

Coslett, H. B., Saffran, E. M., \& Schwoebel, J. (2002). Knowledge of the human body: a distinct semantic domain. Neurology, 59, 357-363.

de Vignemont, F. (2010). Body schema and body image-Pros and cons. Neuropsychologia, 48, 669-680.

Dean, G. M., Dewhurst, S. A., Morris, P. E., \& Whittaker, A. (2005). Selective interference with the use of visual images in the symbolic distance paradigm. Journal of Experimental Psychology. Learning, Memory, and Cognition, 31, 1043-1068.

Eimer, M., Forster, B., Van Velzen, J., \& Prabhu, G. (2005). Covert manual response preparation triggers attentional shifts: ERP evidence for the premotor theory of attention. Neuropsychologia, 43, 957-966.

Felician, O., Ceccaldi, M., Didic, M., Thinus-Blanc, C., \& Poncet, M. (2003). Pointing to body parts: A double dissociation study. Neuropsychologia, 41, 1307-1316.

Fischer, M. H., \& Zwaan, R. A. (2008). Embodied language: A review of the role of the motor system in language comprehension. The Quarterly journal of experimental psychology (Colchester), 61, 825-850.

Flanders, M. (2005). Functional somatotopy in sensorimotor cortex. Neuroreport, 16, 313-316.

Gentner, D. (1977). Children's performance on a spatial analogies task. Child Development, 48, 1034-1039.

Graves, R. (1983). Mouth asymmetry, dichotic ear advantage and tachistoscopic visual field advantage as measures of language lateralization. Neuropsychologia, 21, 641-649.

Hoenig, K., Sim, E. J., Bochev, V., Herrnberger, B., \& Kiefer, M. (2008). Conceptual flexibility in the human brain: Dynamic recruitment of semantic maps from visual, motor, and motion-related areas. Journal of Cognitive Neuroscience, 20, 1799-1814.

Hugdahl, K. (2000). Lateralization of cognitive processes in the brain. Acta Psychologica (Amsterdam), 105, 211-235.

Iachini, S., \& Giusberti, F. (1996). Metric aspects of mental images. Perceptual and Motor Skills, 83, 1243-1250.

Kemmerer, D., \& Tranel, D. (2008). Searching for the elusive neural substrates of body part terms: A neuropsychological study. Cognitive Neuropsychology, 25, 601-629.

Keus, I. M., Jenks, K. M., \& Schwarz, W. (2005). Psychophysiological evidence that the SNARC effect has its functional locus in a response selection stage. Brain Research. Cognitive Brain Research, 24, 48-56.

Kosslyn, S. M., Ball, T. M., \& Reiser, B. J. (1978). Visual images preserve metric spatial information: Evidence from studies of image scanning. Journal of Experimental Psychology. Human Perception and Performance, 4, 47-60.

Laiacona, M., Allamano, N., Lorenzi, L., \& Capitani, E. (2006). A case of impaired naming and knowledge of body parts. Are limbs a separate sub-category? Neurocase, 12, 307-316.

Lobmaier, J. S., \& Mast, F. W. (2007). The Thatcher illusion: Rotating the viewer instead of the picture. Perception, 36, 537-546.

Lopez, C., Bachofner, C., Mercier, M., \& Blanke, O. (2009). Gravity and observer's body orientation influence the visual perception of human body postures. Journal of Vision, 9 (1), 1-14.

Louwerse, M. M., \& Jeuniaux, P. (2008). Language comprehension is both embodied and symbolic. In M. de Vega, A. Glenberg, \& A. C. Graesser (Eds.), Symbols and embodiment: Debates on meaning and cognition (pp. 309-326). Oxford: Oxford University Press.

Louwerse, M. M., \& Jeuniaux, P. (2010). The linguistic and embodied nature of conceptual processing. Cognition, 114, 96-104.

Lu, C. H., \& Proctor, M. L. (1995). The influence of irrelevant location information on performance: A review of the Simon and Spatial Stroop effects. Psychonomic Bulletin Review, 2, 174-207.

Lu, C. H., \& Proctor, R. W. (2001). Influence of irrelevant information on human performance: Effects of S-R association strength and relative timing. The Quarterly Journal of Experimental Psychology (Colchester), 54, 95-136.

Mahon, B. Z., \& Caramazza, A. (2008). A critical look at the embodied cognition hypothesis and a new proposal for grounding conceptual content. Journal of Physiology, Paris, 102, 59-70.

Maki, R. (1981). Categorization and distance effects with spatial linear orders. Journal of Experimental Psychology. Learning, Memory, and Cognition, 7, 15-32.

Marschark, M. (1983). Semantic congruity in symbolic comparisons: Salience, expectancy, and associative priming. Memory \& Cognition, 11, 192-199. 
Marschark, M. \& Paivio, A. (1981). Congruity and the perceptual comparison task. Journal of Experimental Psychology. Human Perception and Performance, 7, 290-308.

Martin, A., Wiggs, C. L., Ungerleider, L. G., \& Haxby, J. V. (1996). Neural correlates of category-specific knowledge. Nature, 379, 649-652.

McGonigle, B., \& Chalmers, M. (1984). The selective impact of question form and input mode on the symbolic distance effect in children. Journal of Experimental Child Psychology, 37, 525-554.

Mishkin, M., \& Forgays, D. G. (1952). Word recognition as a function of retinal locus. Journal of Experimental Psychology, 43, 43-48.

Moyer, R. S., \& Bayer, R. H. (1976). Mental comparison and symbolic distance effect. Cognitive Psychology, 8, 228-246.

Moyer, R. S., \& Landauer, T. K. (1967). Time required for judgements of numerical inequality. Nature, 215, 1519-1520.

New, B., Pallier, C., Brysbaert, M., \& Ferrand, L. (2004). Lexique 2: A new French lexical database. Behavior Research Methods, Instruments, \& Computers, 36, 516-524.

Noordzij, M. L., \& Postma, A. (2005). Categorical and metric distance information in mental representations derived from route and survey descriptions. Psychological Research, 69, 221-232.

Orlov, T., Makin, T. R., \& Zohary, E. (2010). Topographic representation of the human body in the occipitotemporal cortex. Neuron, 68, 586-600.

Paivio, A. (1975). Perceptual comparisons through the mind's eye. Memory \& Cognition, 3, 635-647.

Pecher, D., Zeelenberg, R., \& Barsalou, L. W. (2003). Verifying different-modality properties for concepts produces switching costs. Psychological Science, 14, 119-124.

Pulvermuller, F. (2005). Brain mechanisms linking language and action. Nature Reviews Neuroscience, 6, 576-582.

Pulvermuller, F., \& Fadiga, L. (2010). Active perception: Sensorimotor circuits as a cortical basis for language. Nature Reviews Neuroscience, 11, 351-360.

Reed, C. L., Stone, V. E., Bozova, S., \& Tanaka, J. (2003). The body-inversion effect. Psychological Science, 14, 302-308.

Regard, M., Landis, T., \& Graves, R. (1985). Dissociated hemispheric superiorities for reading stenography vs print. Neuropsychologia, 23, 431-435.

Rueschemeyer, S. -A., Lindemann, O., van Elk, M., \& Bekkering, H. (2009). Embodied cognition: The interplay between automatic resonance and selection-for-action mechanisms. European Journal of Social Psychology, 39, 1180-1187.

Rusconi, E., Pinel, P., Dehaene, S., \& Kleinschmidt, A. (2010). The enigma of Gerstmann's syndrome revisited: A telling tale of the vicissitudes of neuropsychology. Brain, 133, 320-332.

Schmidt, G. L., DeBuse, C. J., \& Seger, C. A. (2007). Right hemisphere metaphor processing? Characterizing the lateralization of semantic processes. Brain and Language, 100, 127-141.
Schwoebel, J., Buxbaum, L. J., \& Coslett, H. B. (2004). Representations of the human body in the production and imitation of complex movements. Cognitive Neuropsychology, 21, 285-298.

Schwoebel, J., \& Coslett, H. B. (2005). Evidence for multiple, distinct representations of the human body. Journal of Cognitive Neuroscience, 17, 543-553.

Segui, J., \& Grainger, J. (1990). Priming word recognition with orthographic neighbors: Effects of relative prime-target frequency. Journal of Experimental Psychology. Human Perception and Performance, 16, 65-76.

Shelton, J. R., Fouch, E., \& Caramazza, A. (1998). The selective sparing of body part knowledge: A case study. Neurocase, 4, 339-351.

Sirigu, A., Grafman, J., Bressler, K., \& Sunderland, T. (1991). Multiple representations contribute to body knowledge processing. Evidence from a case of autotopagnosia. Brain, 114(Pt 1B), 629-642.

Slaughter, V., \& Heron, M. (2004). Origins and early development of human body knowledge. Monographs of the Society for Research in Child Development, 69, vii1-vii102.

Smeets, M. A., Klugkist, I. G., Rooden, S., Anema, H. A., \& Postma, A. (2009). Mental body distance comparison: a tool for assessing clinical disturbances in visual body image. Acta Psychologica (Amsterdam), 132, 157-165.

Struiksma, M. E., Noordzij, M. L., \& Postma, A. (2011). Embodied representation of the body contains veridical spatial information. The Quarterly Journal of Experimenta Psychology (Colchester), 1-14.

Suzuki, K., Yamadori, A., Fuji, T., \& Abe, M. (1997). Category-specific comprehension deficit restricted to body parts. Neurocase, 3, 193-200.

van Dam, W., van Dijk, M., Bekkering, H., \& Rueschemeyer, S. A. (in press). Flexibility in embodied lexical-semantic representations. Hum Brain Mapp.

Verguts, T., Fias, W., \& Stevens, M. (2005). A model of exact small-number representation. Psychonomic Bulletin Review, 12, 66-80.

Weems, S. A., \& Zaidel, E. (2005). The effect of response mode on lateralized lexical decision performance. Neuropsychologia, 43, 386-395.

Westhoff, C., \& Troje, N. F. (2007). Kinematic cues for person identification from biological motion. Perception \& Psychophysics, 69, 241-253.

Witt, A., Cermak, S., \& Coster, W. (1990). Body part identification in 1- to 2-year-old children. American Journal of Occupational Therapy, 44, 147-153.

Zwaan, R. A., \& Yaxley, R. H. (2003a). Hemispheric differences in semantic-relatedness judgments. Cognition, 87, B79-B86.

Zwaan, R. A., \& Yaxley, R. H. (2003b). Spatial iconicity affects semantic relatedness judgments. Psychonomic Bulletin Review, 10, 954-958. 